\title{
Cupins em residências de um bairro em Cáceres, Mato Grosso
}

\section{Termites in residences of a neighbourhood in Cáceres, Mato Grosso}

\author{
Milaine Fernandes dos Santos ${ }^{1 *}$, Carla Cristina Dutra ${ }^{2}$, Arno Rieder ${ }^{3}$, Carla Galbiati ${ }^{3}$
}

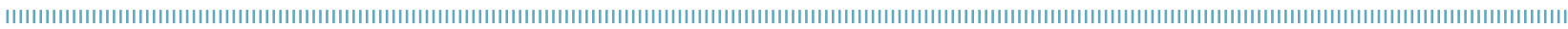

RESUMO: O objetivo deste estudo foi verificar a abundância de cupins e a percepção de moradores sobre a atuação desses insetos como pragas. $\mathrm{O}$ trabalho foi conduzido em quatro residências do bairro Jardim Paraíso, em Cáceres, Mato Grosso. Para isso, foram realizadas inspeções em março, maio, agosto e novembro de 2004 e fevereiro de 2005 por meio de coleta manual (dentro das residências e em árvores nos quintais) e da aplicaçáo de questionários com os moradores para verificar a opinião deles sobre a atuação dos cupins. Foram identificadas quatro espécies: Nasutitermes coxipoensis (48,2\%), Nasutitermes sp. (31\%), Microcerotermes strunckii (7\%) e Heterotermes tenuis (13,7\%). Tanto os gêneros $\left(\chi^{2}=7,880\right.$; $\mathrm{GL}=8 ; \alpha=0,445)$ quanto as espécies $\left(\chi^{2}=20,542 ; \mathrm{GL}=12\right.$; $\alpha=0,058$ ) ocorreram em proporções similares durante o ano, demonstrando que não existe uma relação entre os períodos de coleta e a abundância de insetos. Neste estudo, 50\% dos moradores não consideraram os cupins como pragas em suas residências, provavelmente porque na maioria dos casos esses insetos foram encontrados em árvores. Devido ao baixo número de casas investigadas, mais estudos são necessários para o melhor entendimento sobre a ocorrência de cupins.

PALAVRAS-CHAVE: Heterotermes; Microcerotermes; Nasutitermes; percepçáo; pragas domésticas.

\begin{abstract}
The objective of this study was to evaluate the abundance of termites and the perception of residents about those insects as pests. This study was conducted inside and in the surroundings of four houses in the neighbourhood Jardim Paraíso, in Cáceres, Mato Grosso. Inspections were carried out in March, May, August and November, 2004 and February. 2005, by manual collection (inside the houses and on trees of the backyards). Questionnaires were used to check the opinions of the residents about these insects. Four species were identified: Nasutitermes coxipoensis (48.2\%), Nasutitermes sp. (31\%), Microcerotermes strunckii (7\%) and Heterotermes tenuis $(13.7 \%)$. The genera $\left(\chi^{2}=7.880 ; \mathrm{GL}=8\right.$; $\alpha=0.445)$ and the species $\left(\chi^{2}=20.542 ; \mathrm{GL}=12 ; \alpha=0.058\right)$ occurred in similar proportions. No significant relation was found between the collection periods and the abundance of the insects. In this study, $50 \%$ of the residents did not consider the termites as pests, probably because in most cases $(56.4 \%)$ termites were found only in the trees. Due to the small number of investigated houses, further studies are necessary for a better understanding of the termite incidence.
\end{abstract}

KEYWORDS: Heterotermes; Microcerotermes; Nasutitermes; perception; house pests.

\footnotetext{
'Universidade Federal de Viçosa (UFV) - Viçosa (MG), Brasil.

${ }^{2}$ Universidade Federal da Grande Dourados (UFGD) - Vila Progresso (MS), Brasil.

${ }^{3}$ Universidade do Estado de Mato Grosso (UNEMAT) - Cáceres (MT), Brasil.

*Autor correspondente: milaine.fernandes@gmail.com

Recebido em: 30/10/2013. Aceito em: 21/12/2013.
} 
Algumas espécies de insetos podem ser consideradas pragas quando provocam danos econômicos, sociais, psicológicos ou à saúde do homem (Centro de Vigilância Sanitária, 2000), como é o caso de algumas espécies de cupins (Isoptera) (Constantino, 2002), que causam danos em edificaçôes antigas (Eleotério; Berti-filho, 2000), livros, móveis, roupas (Costa-Leonardo, 2004), árvores, ou, ainda, incômodo pela presença de seus ninhos (Constantino, 2002).

A proliferação de térmitas em áreas urbanas é facilitada pela eliminação de seus competidores e predadores devido a ações antrópicas (WALLER; LA FAGE, 1986), pela grande capacidade que os cupins possuem de explorar recursos alimentares (LimA; Costa-Leonardo, 2007) e pela influência de remanescentes de vegetação nativa, como o cerrado, na composição da fauna local (Constantino; Dianese, 2001). Constantino (2002) mostrou que os cupins-praga ocorrentes em áreas urbanas são menos conhecidos do que os cupins-praga presentes em áreas agrícolas. No entanto, outros autores relataram a presença de cupins com esses hábitos nesses ambientes (FARAGalla; AL Qhtani, 20 i3; Perdereau et al., 2013), sugerindo que estes insetos são amplamente conhecidos e percebidos.

Os cupins vivem predominantemente em regióes com clima quente e ambientes úmidos, sendo a estação chuvosa uma influenciadora na dispersão e nidificação. No Brasil ocorrem aproximadamente 300 espécies, distribuídas entre as famílias Kalotermitidae, Rhinotermitidae, Serritermitidae e Termitidae (Constantino, 1999). A família Rinothermitidae é representada, por exemplo, por Heterotermes Froggat (1896), que atua em vários tipos de hábitat, desde madeiras até ninhos difusos no solo (Constantino, 1999). Dentre os Termitidae, pode-se encontrar cupins com ninhos arborícolas, cartonados ou epígeos (Constantino, 1999), como Nasutitermes Dudley (1890) e Microcerotermes Silvestri (I90 I).

Heterotermes, Nasutitermes e Microcerotermes possuem distribuição pantropical, sendo que os dois primeiros são encontrados em todas as regiôes do Brasil, e o último na Amazônia e no cerrado. Entretanto, Microcerotermes não apresenta distribuição precisa de espécies (Constantino, 1999). Algumas espécies pertencentes a esses gêneros habitam áreas urbanas, como Heterotermes tenuis Hagen (1858) (Constantino; Dianese, 2001), Nasutitermes coxipoensis Holmgren (1910) (LAFFonT et al., 2004), Nasutitermes sp. (Mello, 2011) e Microcerotermes strunckii Sörensen (1884) (LAFFONT et al., 2004).

De acordo com Constantino (2002), M. strunckii e $H$. tenuis sáo consideradas pragas estruturais de menor importância, sendo que $H$. tenuis é tida como praga agrícola de mais importância em cultivos de cana-de-açúcar e eucalipto. Apesar disso, a maioria das espécies de cupim não acarreta prejuízos ao homem, pois são imprescindíveis à manutençấo dos processos de decomposiçáa e para os fluxos de carbono e nutrientes no solo (Bandeira; VAsConcellos, 2002).

Um diagnóstico sobre pragas de importância doméstica inclui os cupins entre as mais citadas em Cáceres (MT)
(RIEDER, 1987), portanto, a realização de estudos com esse enfoque é fundamental para averiguar a existência de possíveis danos econômicos. Nosso objetivo foi verificar a abundância de cupins ao longo do ano e a percepçáo de moradores sobre a atuação desses insetos como pragas em Cáceres (MT).

Essa pesquisa é parte do projeto "Pragas Domésticas em Cáceres" (PDC), conduzido em quatro residências do bairro Jardim Paraíso (JP). As coletas de cupins foram realizadas em março, maio, agosto e novembro de 2004 e fevereiro de 2005 em ambientes internos e externos das residências. Durante as inspeçóes, foram procuradas evidências da presença de cupins subterrâneos e/ou de madeira seca. Os túneis escavados na madeira foram considerados a principal evidência da presença de cupins subterrâneos. Quanto aos cupins de madeira seca, foi observada a existência de orifícios e grânulos fecais na superfície de móveis e outras estruturas de madeira.

As espécies de cupins foram identificadas por especialista da Universidade Federal de Viçosa (MG), sendo que as amostras foram depositadas nesta mesma instituiçăo, bem como na coleção do projeto Pragas Domésticas, da Universidade do Estado de Mato Grosso.

A relação entre a abundância das espécies de cupins durante os meses de coleta foi avaliada por meio do teste do qui-quadrado $\left(\chi^{2}\right)$, ao nível de $5 \%$ de significância. Os dados foram analisados por meio do programa SPSS para Windows.

Nesta pesquisa, foram identificadas 29 amostras em termos de espécie. Entre elas, encontramos Heterotermes (13,7\%), Nasutitermes (79,1\%) e Microcerotermes (7\%). A abundância desses gêneros foi observada em proporções semelhantes durante os meses do ano $\left(\chi^{2}=7,880 ; \mathrm{GL}=8 ; \alpha=0,445\right)$. As espécies encontradas foram $N$. coxipoensis (48,2\%), Nasutitermes sp. (31\%), M. strunckii (7\%) e H. tenuis (13,7\%). A abundância dessas espécies também foi encontrada em proporções semelhantes durante os meses do ano $\left(\chi^{2}=20,542 ; \mathrm{GL}=12 ; \alpha=0,058\right)$.

$N$. coxipoensis náo foi encontrada em novembro e fevereiro, sendo mais abundante em março (27,6\%); Nasutitermes sp. não ocorreu em março, contudo, foi a espécie mais frequente durante o ano, em maio $(6,9 \%)$, agosto $(3,4 \%)$, novembro $(10,3 \%)$ e fevereiro (10,3\%); M. strunckii ocorreu apenas em novembro e fevereiro, com frequência igualada de 3,5\%; Já $H$. tenuis ocorreu em março $(3,4 \%)$, novembro $(3,4 \%)$ e fevereiro do ano seguinte $(6,9 \%)$ (Tabela 1$)$. Não foram encontrados cupins de madeira seca nos locais inspecionados.

N. coxipoensis $(48,2 \%)$ e Nasutitermes sp. (31\%) foram mais frequentes nas residências (Tabela 1). Esse gênero apresenta ampla distribuição entre os habitats (Constantino, 1999), e provavelmente por este motivo verificamos elevada abundância dessas espécies neste estudo. Nasutitermes foi encontrado causando danos em armários, cadeiras, estantes, guarda-roupas, janelas e mesas de residências na Paraíba (MelLo, 2011), assim como em paredes de alvenaria e telhados em Pernambuco, o que pode indicar maior capacidade de adaptação deste gênero aos processos de urbanização e às modificaçôes do ecossistema natural (Moraes et al., 2007). Faragalla; Al Qhtani (2013) também mostraram que 
cupins Termitidae podem ser encontrados em áreas urbanas. M. strunckii ocorreu em apenas duas coletas, contudo, podem ocasionar danos nas residências devido às potencialidades de infestação, principalmente em árvores (Constantino, 1999). Constantino (2002) e Laffont et al. (2004) também encontraram $M$. strunckii em áreas urbanas.

Os cupins com hábitos subterrâneos apresentam ampla dispersão e podem infestar árvores, paredes de alvenaria, pisos, tijolos (Berti-filho; Fontes, 1995). Tem-se como exemplo de cupins subterrâneos a espécie $H$. tenuis, frequentemente encontrada em plantaçóes, causando danos à cana-de-açúcar e ao milho (ZoRZENON, 2002).

A abundância de cupins foi maior em árvores (56,4\%), especificamente em Anacardium occidentale L (47,8\%) e Mangifera indica L (8,6\%) (Tabela 2). De acordo com Constantino (1999) e LafFont et al. (2004), M. strunckii e N. coxipoensis comumente são encontradas no solo e em árvores, o que explica nossos resultados devido a ocorrência destas espécies em árvores nas residências inspecionadas. Apesar de encontrarmos baixa abundância $(4,4 \%)$ de $H$. tenuis, outros estudos mostram a ocorrência desta espécie em áreas urbanas (LAFFONT et al., 2004) provocando danos em árvores (Brazolin et al., 2010). Nesta pesquisa, também foram encontrados cupins causando danos em objetos de madeira (26\%) e parede (17,6\%) (Tabela 2). Dessa forma, a variedade de locais para nidificação e abrigo em áreas urbanas facilitam a dispersão de cupins devido à oferta de celulose, o que compromete a sua sanidade, causando prejuízos aos moradores.

Verificamos que $50 \%$ dos moradores qualificaram os cupins como pragas, ao contrário dos outros $50 \%$. Segundo Eleotério; Berti Filho (2000), o conhecimento por parte da populaçáo sobre a problemática dos cupins em áreas urbanas ainda é escasso, e isso pode ter influenciado a opiniáo dos entrevistados. Dessa forma, recomendamos o desenvolvimento de outras pesquisas com este enfoque para mais esclarecimentos, já que o risco de infestação aumenta com a idade dos imóveis (Eleotério; Berti Filho, 2000), e, nesse sentido, a opinião dos moradores também pode mudar. Estudos biológicos sobre os cupins podem ainda contribuir para a adoção de medidas alternativas de controle, uma vez que inseticidas sáo os métodos mais utilizados pela população (NAscimento et al., 2007).

A abundância de cupins entre os locais foi similar, estando principalmente relacionada à presença de árvores nos quintais, o que favoreceu a ocorrência de cupins com hábitos arborícolas. A porcentagem de entrevistados que qualificaram os cupins como pragas $(50 \%)$ pode estar relacionada com o grau de conhecimento por parte desses moradores sobre a atuação dos cupins nas residências. Embora nossos resultados sejam preliminares, considerando a amostragem, ainda assim podem ajudar a esclarecer a distribuição dessas espécies em residências ao longo do ano, contribuindo para a adoção de medidas preventivas.

\section{AGRADECIMENTOS}

Ao professor Og Francisco Fonseca de Souza, do Departamento de Biologia Animal da Universidade Federal de Viçosa (UFV), pela identificação taxonômica dos cupins, e à professora Maria Antônia Carniello, do Departamento de Ciências Biológicas da Universidade do Estado de Mato Grosso (UNEMAT), pela identificação das espécies vegetais envolvidas neste estudo.

Tabela 1. Abundância de cupins em quatro residências durante um ano em Cáceres (MT) entre 2004 e 2005.

\begin{tabular}{|c|c|c|c|c|}
\hline \multirow{2}{*}{ Meses de coleta } & \multicolumn{4}{|c|}{ Espécies (ocorrências ) } \\
\hline & N. coxipoensis & Nasutitermes sp. & M. strunckii & H. tenuis \\
\hline mar/04 & $8(27,6 \%)$ & $\mathrm{O}(0 \%)$ & $\mathrm{O}(0 \%)$ & $1(3,4 \%)$ \\
\hline mai/04 & $3(10,3 \%)$ & $2(6,9 \%)$ & $\mathrm{O}(0 \%)$ & $\mathrm{O}(0 \%)$ \\
\hline ago/04 & $3(10,3 \%)$ & $1(3,4 \%)$ & $\mathrm{O}(0 \%)$ & $\mathrm{O}(0 \%)$ \\
\hline nov/04 & $\mathrm{O}(0 \%)$ & $3(10,3 \%)$ & $1(3,5 \%)$ & $1(3,4 \%)$ \\
\hline fev/05 & $\mathrm{O}(0 \%)$ & $3(10,3 \%)$ & $1(3,5 \%)$ & $2(6,9 \%)$ \\
\hline \multirow{2}{*}{ Total } & $14(48,2 \%)$ & $9(31 \%)$ & $2(7 \%)$ & $4(13,7)$ \\
\hline & \multicolumn{4}{|c|}{$29(100 \%)$} \\
\hline
\end{tabular}

Tabela 2. Locais de ocorrência de cupins em quatro residências em Cáceres (MT) entre 2004 e 2005.

\begin{tabular}{|c|c|c|c|c|}
\hline \multirow{2}{*}{ Locais de ocorrência } & \multicolumn{4}{|c|}{ Espécies } \\
\hline & N. coxipoensis & Nasutitermes sp. & M. strunckii & H. tenuis \\
\hline Anacardium occidentale & $13 \%$ & $21,7 \%$ & $8,7 \%$ & $4,4 \%$ \\
\hline Mangifera indica & $8,6 \%$ & - & - & - \\
\hline Objetos de madeira & $13 \%$ & $8,7 \%$ & - & $4,4 \%$ \\
\hline Parede & $8,8 \%$ & $4,4 \%$ & - & $4,4 \%$ \\
\hline Frequência parcial & $43 \%$ & $34,8 \%$ & $8,7 \%$ & $13,2 \%$ \\
\hline Frequência total & \multicolumn{4}{|c|}{$100,0 \%$} \\
\hline
\end{tabular}




\section{REFERÊNCIAS}

BANDEIRA, A.G.; VASCONCELLOS, A.A quantitative survey of termites in a gradient of disturbed highland forest in Northeastern Brazil (Isoptera). Sociobiology, v.39, p.429- 439, 2002.

BERTI-FLHO, E.; FONTES, L.R. Biologia e controle de cupins. Piracicaba: FEALQ. 1995. 184p.

BRAZOLIN, S.; TOMAZELLO FILHO, M.; AMARAL, R.D.A.M.; OLIVEIRA NETO, M.A. Associação entre fungos apodrecedores e cupins subterrâneos no processo de biodeterioração do lenho de árvores de Tipuana tipu (Benth.) O. Kuntze da cidade de São Paulo, SP. Scientia Forestalis, Piracicaba, v.38, n.86, p.215-224, 2010.

CVS. CENTRO DE VIGILÂNCIA SANITÁRIA. Norma técnica para empresas prestadoras de serviço em controle de vetores e pragas urbanas. Lei Estadual no 10.083, de 23 de setembro de 1998. Resolução RDC $n^{\circ} 18$, de 29 de fevereiro de 2000. Portaria n 9, 2000. p1-29.

CONSTANTINO, R. Chave ilustrada para identificação dos gêneros de cupins (Insecta: Isoptera) que ocorrem no Brasil. Papéis Avulsos de Zoologia, São Paulo, v.40, n.25, p.387-448, 1999.

CONSTANTINO, R. The pest termites of South America: taxonomy, distribution and status. Journal of Applied Entomology, Berlim, v. 126, p.355-365, 2002.

CONSTANTINO, R.; DIANESE, E.C. The urban termite fauna of Brasília, Brazil. Sociobiology, v.38, n.3, p.323-326, 2001.

COSTA-LEONARDO, A.M. Pragas atacam madeira e móveis. Revista da Madeira, Curitiba, ano 14, n.82, 2004.

ELEOTÉRIO, E.S.R.; BERTI FILHO, E. Levantamento e identificação de cupins (Insecta: Isoptera) em área urbana de Piracicaba - SP. Ciência Florestal, Santa Maria, v.10, n. 1, p.125-139, 2000.

FARAGALLA, A.R.A.; AL QHTANI, M.H. The urban termite fauna (Isoptera) of Jeddah city, western Saudi Arabia. Life Science Journal, New York, v. 10, n.4, p. 1695-1701, 2013.
LAFFONT, E.R.; TORALES, G.J.; CORONEL, J.M.; ARBINO, M.O.; GODOY, M.C. Termite (Insecta, Isoptera) fauna from National Parks of the northeast region of Argentina. Scientia Agricola, Piracicaba, v.61, n.6, p.665-670, 2004.

LIMA, J.T.; COSTA-LEONARDO, A.M. Recursos alimentares explorados pelos cupins (Insecta: Isoptera). Biota Neotropica, Campinas, v.7, n.2, p.243-250, 2007.

MELLO, A.P. Ataque de cupins (Isoptera) em residências do município de Fagundes/PB, nordeste do Brasil. In: X CONGRESSO DE ECOLOGIA DO BRASIL, 201 1, São Lourenço. Anais. São Lourenço: Sociedade de Ecologia do Brasil, 2011. Versão eletrônica.

MORAES, F.M.; OLIVEIRA, F.M.; MATIAS, G.R.R.S.; ALBUQUERQUE, A.C. Termitofauna (Cupins: Isoptera), nos bairros Várzea e Casa Amarela em uma área urbana de Recife, PE. Biológico, São Paulo, v.69, n.2, p.113-198, 2007.

NASCIMENTO, W.C.; GONÇALVES, C.A.; SOARES, N.S.; ALVES, F.A.A.; ALMEIDA M.P. Etnoconhecimento sobre o controle de cupins nas residências de Itumbiara-GO. In: VIII CONGRESSO DE ECOLOGIA DO BRASIL, 2007, Caxambu. Anais. Caxambu: Sociedade de Ecologia do Brasil, 2007. Versão eletrônica.

PERDEREAU, E.; VELONÀ, A; DUPONT, S.; LABÉDAN, M. Colony Breeding Structure of the Invasive Termite Reticulitermes urbis (Isoptera: Rhinotermitidae). Journal of Economic Entomology, Annapolis, v.106, n.5, p.2216-2224, 2013.

RIEDER, A. Pragas de fundo de quintal no meio urbano. Cuiabá: EMATER-MT, 1987. 36p.

WALLER, D.A.; LA FAGE, J.P. Nutritional ecology of termites. In: Nutritional ecology of insects, mites, spiders and related invertebrates. New York: Wiley-Interscience Publication, 1986. p.487-532.

ZORZENON, F.J. Noções sobre as principais pragas urbanas. Biológico, São Paulo, v.64, n.2, p.231-234, 2002. 